\title{
Risk factors and patterns of lymph node involvement in primary gastric large B-cell lymphoma: implications for target definition
}

This article was published in the following Dove Press journal:

OncoTargets and Therapy

29 July 2016

Number of times this article has been viewed

\section{Ximei Zhang \\ Peiguo Wang \\ Lujun Zhao \\ Zhiyong Yuan \\ Ping Wang}

Department of Radiation Oncology, Tianjin's Clinical Research Center for Cancer and Key Laboratory of Cancer Prevention and Therapy, Tianjin Medical University Cancer Institute and Hospital, National Clinical Research Center for Cancer, People's Republic of China
Correspondence: Ximei Zhang Department of Radiation Oncology, Tianjin's Clinical Research Center for Cancer and Key Laboratory of Cancer Prevention and Therapy, Tianjin Medical University Cancer Institute and Hospital, National Clinical Research Center for Cancer, Huanhuxi Road, Hexi District, Tianjin 300060, People's Republic of China

Tel +86 22 2334 I 405

Fax +86 222334 I 405

Email xiangrikuigirl@।26.com
Background: The aim of this study was to identify the appropriate radiation volume for primary gastric diffuse large B-cell lymphoma (PG-DLBCL).

Methods: We retrospectively analyzed the clinical and pathological findings of 68 patients treated with total gastrectomy and D2 lymphadenectomy.

Results: There were 23, 14, and 29 patients with stage I, stage II, and stage IIE disease, respectively, and 30 patients had lymph node involvement. Primary tumor location, as well as the depth of invasion, was significantly associated with lymph node involvement. When the tumor was limited to the muscularis, the involved lymph nodes were found to be perigastric nodes. For tumors invading beyond the muscularis, regional lymph nodes were involved.

Conclusion: The optimal radiation volume for patients with PG-DLBCL is largely dependent on the primary location and depth of invasion. Larger series and longer follow-up are needed to further delineate the radiation volumes for PG-DLBCL.

Keywords: primary gastric lymphoma, failure patterns, radiotherapy, chemotherapy, prognosis

\section{Introduction}

In the last two decades, surgical treatment of primary gastric lymphomas (PGL) has largely been abandoned since good long-term results can be obtained with organ-sparing treatment while avoiding post-gastrectomy morbidity and mortality. ${ }^{1-3}$ Nowadays, gastrectomy is considered only in the cases of hemorrhage, perforation, or obstruction. Stomach-preserving treatment with chemotherapy, sometimes in conjunction with involved-site radiotherapy (ISRT), is becoming the therapy of choice for PGL. ${ }^{4-6}$ In addition, Helicobacter pylori-related diffuse large B-cell lymphoma (DLBCL) of the stomach might be potentially treated via $H$. pylori eradication therapy alone. ${ }^{7}$

Primary gastric DLBCL (PG-DLBCL) comprises $40 \%-70 \%$ of all primary gastric non-Hodgkin lymphomas. ${ }^{8}$ The role of radiotherapy in PG-DLBCL is still being debated. However, data from a retrospective series showed that irradiation significantly reduced local relapse rate. ${ }^{9}$ In addition, a prospective study also demonstrated favorable outcomes. ${ }^{6}$ There does not seem to be any reason to treat PG-DLBCL differently from nodal diffuse large B-cell lymphoma because these malignancies share the same phenotypic and biological characteristics. ${ }^{10}$ As a result, chemotherapy followed by consolidative radiotherapy has been used by many physicians as a primary therapy. ${ }^{11}$ However, a consensus regarding the appropriate volume for irradiating gastric lymphoma has not been reached. Different targets have been employed ranging from the whole abdomen to the recently described involved-field radiotherapy and ISRT. ${ }^{12}$ 
When using involved-field radiotherapy, whether to include perigastric lymph nodes or regional nodes in the treatment of PG-DLBCL is unclear.

To clearly identify the appropriate treatment volume in PG-DLBCL, we retrospectively analyzed the clinical and pathological findings of a relatively large patient series treated with total gastrectomy and D2 lymphadenectomy.

\section{Methods and materials}

Between 2008 and 2012, a total of 68 patients diagnosed with PG-DLBCL were treated with gastrectomy at Tianjin Medical Cancer Center. Some of the patients were indicated for primary surgery for the prevention of severe bleeding, perforation, occlusion, or bulky tumors secondary to rapid tumor growth; others were misdiagnosed as having gastric cancer prior to surgery. The diagnosis was confirmed by pathologic and immunohistochemical studies according to the World Health Organization classification of lymphoid tissues. ${ }^{13}$ Immunohistochemical staining and scoring for CD10, BCL-6, and MUM-1 were performed on formalin-fixed paraffin-embedded tissues, and then the patients were assigned as germinal center B-cell-like (GCB) phenotype or non-GCB phenotype using the algorithm of Hans et al. ${ }^{14}$ All the patients were diagnosed with large B-cell lymphoma and none with mucosa-associated lymphoid tissue (MALT) lymphoma. The patients were staged according to the Lugano staging system for gastrointestinal lymphomas, ${ }^{15}$ and pretreatment evaluation included computed tomography (CT) of the chest, abdomen, and pelvis; endoscopic ultrasonography; and bone marrow examination.

Given the evidence that PG-DLBCL is always multicentric, all the patients underwent radical gastrectomy and D2 lymphadenectomy even when the tumor appeared to be confined to one area. The average number of resected lymph nodes was 18 (range: 13-58). Chemotherapy and radiotherapy were used as adjuvant treatment after surgery. All the patients except three underwent surgery combined with four to six cycles of adjuvant chemotherapy using standard CHOP or $\mathrm{CHOP}-$ like regimens. In view of the encouraging results of rituximab for nodal DLBCL, 26 patients in this study received RCHOP, and patients who could not afford rituximab were prescribed the CHOP regimen. Twenty patients with multiple lymph node involvement or bulky disease also received radiation on the gastric bed and regional lymph node regions with a radiation dose of 36-40 Gy at 2 Gy per fraction.

We reviewed the pathological records including the infiltration depth of lymphoma and the number and the stations of involved lymph nodes in order to understand the patterns of tumor spread and lymph node involvement. In addition, patterns of failure for patients with PG-DLBCL were also analyzed. This study was approved by the institutional review board of Cancer Institute and Hospital, Tianjin Medical University, and all the patients provided written informed consent in accordance with the Declaration of Helsinki.

\section{Statistical analysis}

Local failure was defined as disease progression or recurrence at the primary site, independent of the involvement of distant lymph nodes and extranodal sites. Categorical data between groups and associations between lymph node involvement and clinical characteristics were compared using the chi-square analysis.

\section{Results}

Table 1 shows the clinicopathologic characteristics of patients with PG-DLBCL. The study included 36 male and

Table I Clinical characteristics of patients with PG-DLBCL

\begin{tabular}{|c|c|c|}
\hline Characteristics & No & $\%$ \\
\hline \multicolumn{3}{|l|}{ Sex } \\
\hline Male & 36 & 52.9 \\
\hline Female & 32 & 47.1 \\
\hline \multicolumn{3}{|l|}{ Age (years) } \\
\hline Median (range) & $57(19-78)$ & \\
\hline$<60$ & 44 & 64.7 \\
\hline$\geq 60$ & 24 & 35.3 \\
\hline \multicolumn{3}{|l|}{ Stage } \\
\hline I & 25 & 36.8 \\
\hline$\|$ & 14 & 20.6 \\
\hline IIE & 29 & 42.6 \\
\hline \multicolumn{3}{|c|}{ Lymph node involvement } \\
\hline Positive & 30 & 44.1 \\
\hline Negative & 38 & 55.9 \\
\hline \multicolumn{3}{|l|}{ Size $(\mathrm{n}=66 ; \mathrm{cm})$} \\
\hline$<10$ & 20 & 30.3 \\
\hline $10-20$ & 37 & 56.1 \\
\hline$\geq 20$ & 9 & 13.6 \\
\hline \multicolumn{3}{|l|}{ Subtypes $(n=55)$} \\
\hline $\mathrm{GCB}$ & 13 & 23.6 \\
\hline$A B C$ & 42 & 76.4 \\
\hline \multicolumn{3}{|l|}{ Site } \\
\hline Cardia & 5 & 10.4 \\
\hline Body & 24 & 29.2 \\
\hline Antrum & 30 & 47.9 \\
\hline Full stomach & 9 & 12.5 \\
\hline \multicolumn{3}{|l|}{ Depth of infiltration } \\
\hline Mucosa & 7 & 10.3 \\
\hline Submucosa & 9 & 13.2 \\
\hline Muscular & 12 & 17.6 \\
\hline Serosa & 30 & 44.2 \\
\hline Adjacent organs & 10 & 14.7 \\
\hline
\end{tabular}

Abbreviations: PG-DLBCL, primary gastric diffuse large B-cell lymphoma; No, number; $G C B$, germinal center $B$-cell-like; $A B C$, activated B-cell-like. 
32 female patients with a median age of 57 years (range: 19-78 years). According to the Lugano staging system, 25, 14, and 29 patients had stage I, II, and IIE disease, respectively, and 30 patients had lymph node involvement. For evaluating tumor size, the Revised Response Evaluation Criteria in Solid Tumors (version 1.1) ${ }^{16}$ instead of the maximal tumor diameter was applied to assess the tumor burden. Accordingly, 37 and 9 patients had tumors greater than 10 and $20 \mathrm{~cm}$, respectively. With respect to the primary location, the gastric antrum was the most commonly involved site, followed by the gastric body, whole stomach, and cardia. PG-DLBCL tends to invade aggressively, therefore the majority of the patients had penetration into the serosa and adjacent organs.

To identify the factors affecting lymph node involvement, the associated clinicopathologic characteristics were analyzed and shown in Table 2 . The results demonstrated that neither age nor sex was relevant to lymph node involvement. The lymph node involvement rate increased with an increase

Table 2 Factors associated with lymph node involvement in patients with PG-DLBCL

\begin{tabular}{|c|c|c|}
\hline Characteristics & $\begin{array}{l}\text { Positive lymph } \\
\text { node rate } \%\end{array}$ & $P$-value \\
\hline Sex & & 0.584 \\
\hline Male & $37.5(13 / 32)$ & \\
\hline Female & $47.2(17 / 36)$ & \\
\hline Age (years) & & 0.417 \\
\hline$<60$ & $47.7(2 \mathrm{I} / 44)$ & \\
\hline$\geq 60$ & $37.5(9 / 24)$ & \\
\hline Stage & & 0.000 \\
\hline I & $0(0 / 25)$ & \\
\hline II & $100(14 / 14)$ & \\
\hline IIE & $55.2(16 / 29)$ & \\
\hline Size $(\mathrm{cm})$ & & 0.280 \\
\hline$<10$ & $35.0(7 / 20)$ & \\
\hline $10-20$ & $43.2(16 / 37)$ & \\
\hline$\geq 20$ & $66.7(6 / 9)$ & \\
\hline Subtypes & & 0.224 \\
\hline GCB & $30.8(4 / 13)$ & \\
\hline$A B C$ & $50.0(2 \mathrm{I} / 42)$ & \\
\hline Site & & 0.005 \\
\hline Cardia & $60.0(3 / 5)$ & \\
\hline Body & $62.5(15 / 24)$ & \\
\hline Antrum & $20.0(6 / 30)$ & \\
\hline Full stomach & $66.7(6 / 9)$ & \\
\hline Depth of infiltration & & 0.004 \\
\hline Mucosa & $0(0 / 7)$ & \\
\hline Submucosa & $22.2(2 / 9)$ & \\
\hline Muscular & $16.7(2 / 12)$ & \\
\hline Serosa & $63.3(19 / 30)$ & \\
\hline Adjacent organs & $70.0(7 / / 0)$ & \\
\hline
\end{tabular}

Abbreviations: PG-DLBCL, primary gastric diffuse large B-cell lymphoma; $\mathrm{GCB}$, germinal center B-cell-like; $\mathrm{ABC}$, activated B-cell-like. in tumor size, although the association was not significant. We also found that primary location was significantly associated with lymph node involvement. The rate was rather low when the gastric antrum was involved, whereas when the whole stomach was involved, the rate approached $70 \%$.

It is of note that the lymph node involvement rate increased with the depth of invasion into the stomach. When the tumor was limited to the mucosa, there was no lymph node involvement, but patients with tumor invasion into serosa and adjacent organs had a lymph node involvement rate of around $60 \%$. Two out of 12 patients with muscularis invasion had lymph node involvement and the involved lymph nodes were confined to the perigastric region. Figure 1 shows the locations of lymph node involvement in cases with mucosa and muscularis invasion.

In patients with serosa invasion, 30 out of 68 patients had lymph node involvement. Among them, 12 patients also had second-echelon (left gastric artery, common hepatic artery, and celiac nodes) and seven patients had third-echelon (hepatoduodenal ligament and superior mesenteric vessels nodes) lymph node involvement except for the perigastric lymph nodes. The seven patients with third-echelon involvement also had other poor prognostic features. They all had a tumor size greater than $10 \mathrm{~cm}$ and had the activated B-cell-like subtype. For those tumors involved with adjacent organs, $70 \%(7 / 10)$ had lymph node involvement. In these cases, the involved lymph nodes resided in the second-echelon region, mainly involving the left gastric artery, common

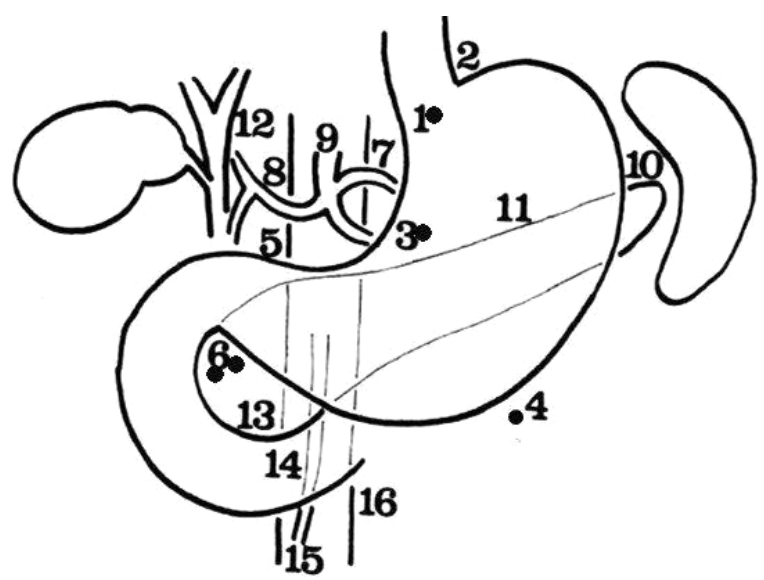

Figure I Locations of lymph node involvement in patients with PG-DLBCL mucosa and muscularis involvement.

Notes: Dots mean the number of involved lymph nodes. The lymph node stations are numbered according to the Japanese Research Society for Gastric Cancer: I, right cardiac; 2 , left cardiac; 3 , lesser curvature; 4 , greater curvature; 5 , suprapyloric; 6 , infrapyloric; 7, left gastric artery; 8, common hepatic artery; 9, celiac artery; 10, splenic hilum; II, splenic artery; 12, hepatoduodenal ligament; 13, retropancreatic; 14, mesenteric root; 15, middle colic artery; 16, paraaortic nodes.

Abbreviation: PG-DLBCL, primary gastric diffuse large B-cell lymphoma. 


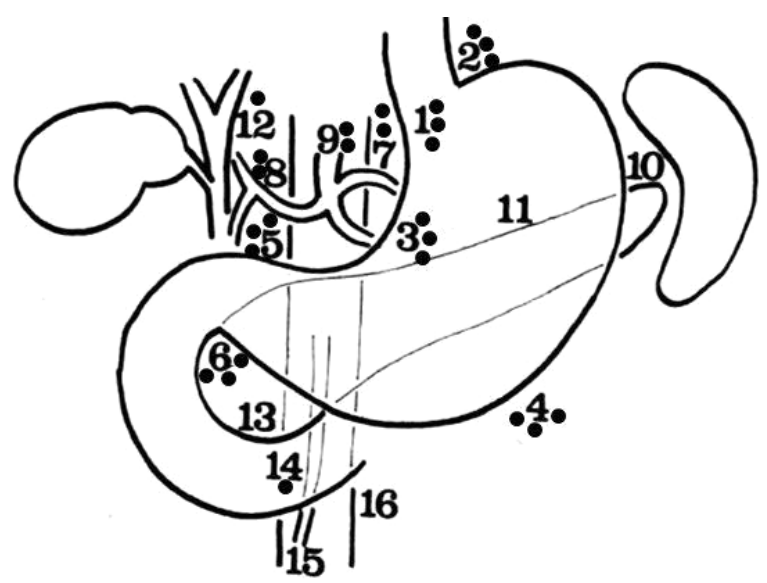

Figure 2 Locations of lymph node involvement in patients with PG-DLBCL serosa and adjacent organ involvement.

Notes: Dots mean the number of involved lymph nodes. The lymph node stations are numbered according to the Japanese Research Society for Gastric Cancer: I, right cardiac; 2, left cardiac; 3 , lesser curvature; 4, greater curvature; 5 , suprapyloric; 6, infrapyloric; 7, left gastric artery; 8, common hepatic artery; 9, celiac artery; 10, splenic hilum; II, splenic artery; 12, hepatoduodenal ligament; 13, retropancreatic; 14, mesenteric root; 15, middle colic artery; 16, paraaortic nodes.

Abbreviation: PG-DLBCL, primary gastric diffuse large B-cell lymphoma.

hepatic artery, and celiac nodes. The locations of lymph node involvement are shown in Figure 2.

We also analyzed the lymph node involvement rate according to different subtypes. Patients with GCB-type DLBCL had $30.8 \%$ lymph node involvement, whereas patients with activated B-cell-like type DLBCL had 50.0\% lymph node involvement. There was no significant difference between the two groups.

With a median follow-up of 45 months, 18 patients developed progressive disease or relapse. The patterns of

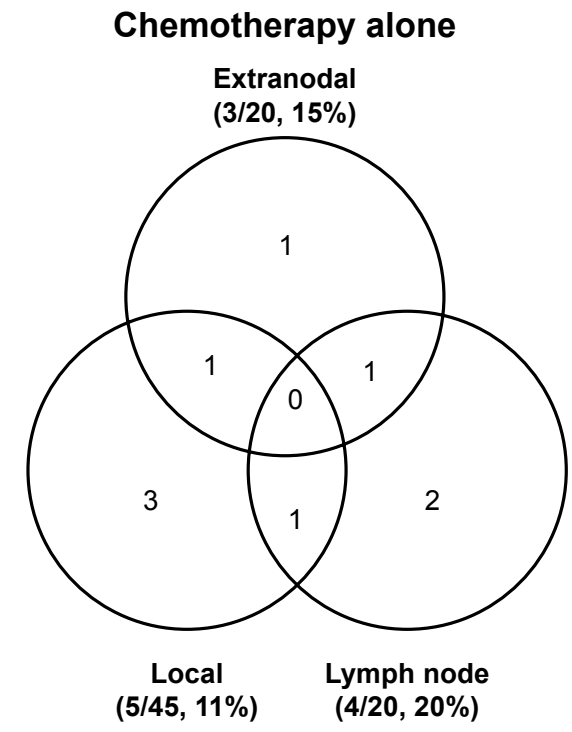

Figure 3 Comparisons of the patterns of treatment failure in the two groups. treatment failure in the two groups were compared (Figure 3). Among them, five patients $(5 / 45,11.1 \%)$ in the chemotherapy-alone group experienced local failure, whereas none of the 20 patients $(0 / 20,0)$ in the combined modality group developed local relapse $(P=0.121)$. Progressive lymph node involvement rates were comparable between the treatment groups ( $4 / 20$ vs $3 / 45, P=0.109)$. Extranodal dissemination was observed in four patients $(4 / 45,8.9 \%)$ in the combined group and three patients $(3 / 20,15 \%)$ in the chemotherapy group $(P=0.463)$. Patients with surgery alone experienced not only local but also lymphatic recurrence in the head and neck area, retroperitoneum, and spleen. The acute and late complications of radiotherapy were acceptable. The majority of the patients had grade 0-2 acute toxicities, and no renal toxicity was observed in these patients.

\section{Discussion}

Treatment of patients with PG-DLBCL is quite unclear. Surgery has played a diminishing role in the treatment of PG-DLBCL in recent years since improved outcomes of conservative treatment have been reported. ${ }^{3}$ Recently, systemic chemotherapy with or without radiotherapy is the preferred treatment for PG-DLBCL. The role of consolidative radiotherapy after chemotherapy has been demonstrated in several prospective randomized controlled trials for nodal DLBCL. ${ }^{17-20}$ A systematic review conducted by Santos et al showed that radiation could enhance progression-free survival after chemotherapy. ${ }^{21}$ Therefore, since nodal DLBCL and PG-DLBCL share many of the same biological characteristics, systemic chemotherapy followed by radiotherapy

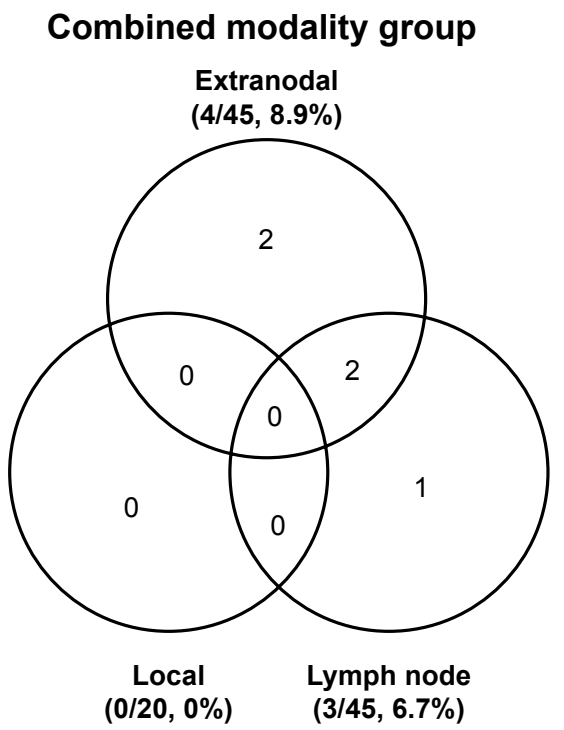


is now the preferred treatment for PG-DLBCL in certain centers. Evidence from retrospective data also confirmed that the addition of radiation significantly reduced the local relapse rate compared with chemotherapy alone. ${ }^{9}$

An important issue for the use of radiotherapy is the severe acute and late side effects that can be caused by irradiation. However, the side effects seem to be acceptable., ${ }^{4,22}$ More recently, the wide use of modern radiation techniques such as intensity-modulated radiotherapy (IMRT) is expected to reduce the doses to organs at risk, therefore decreasing the risk of acute and late effects. Multiple reports on gastric cancer have confirmed the preponderance of IMRT over conventional radiotherapy. ${ }^{23-26}$ Although there is little direct evidence on gastric lymphoma, patients with PG-DLBCL would be expected to benefit from IMRT. Radiotherapy in this study was well tolerated, and only mild gastrointestinal toxicity or renal toxicity was observed. This is consistent with the published literature.

To clearly specify the optimal radiation field for PGL, we should figure out the patterns of lymph node involvement in PGL. Several studies have described the factors associated with lymph node involvement and the locations of lymph nodes. Montalban et al reported that the lymph node involvement rate in low- and high-grade MALT was $26 \%$ and $65 \%$, respectively. ${ }^{27}$ Eidt et al found that the rate of lymph node involvement was $22 \%$ in MALT patients with penetration into the mucosa or submucosa and $63 \%$ in those with infiltration above the muscular layer. ${ }^{28}$ Park et al performed a detailed analysis of the characteristics associated with lymph node involvement in patients with MALT. ${ }^{29}$ However, all the aforementioned studies demonstrated the influencing factors associated with lymph node involvement in MALT. To date, no studies have been conducted on PG-DLBCL.

According to our study, the depth of invasion and tumor location were significantly associated with lymph node involvement. Only $44.1 \%$ of our patients had lymph node involvement, which is lower than that reported in other series, partly because most of our tumors were located in the antrum and tumors in the antrum have the lowest rate of lymph node involvement. This finding is consistent with the conclusions in the study by Park et al. ${ }^{29} \mathrm{We}$ also found that there were differences in the distribution of involved lymph nodes. When the tumor was contained within the muscularis propria, the involved lymph nodes were all perigastric nodes. For tumors infiltrating beyond the muscularis, the involved lymph nodes were regional nodes, including the third-echelon lymph nodes.
Based on these findings, we believe that when tumors infiltrate within the muscularis, the whole stomach and perigastric adenopathy are the appropriate radiation volume, whereas when tumors infiltrate beyond the muscle layer, the optimal volume includes the stomach and regional lymph nodes. There is no study regarding the best time to perform radiation in the rituximab era; however, we believe that four to six cycles of RCHOP followed by involved-field radiotherapy or ISRT is the treatment of choice, especially for patients with residual tumor after chemotherapy.

The primary pattern of failure for patients with PG-DLBCL was local relapse. Patients who underwent adjuvant radiotherapy had no local relapses, whereas those who did not receive radiotherapy had a high rate of disease progression. Although randomized trial data are unavailable because of the rarity of the disease, the difference in our study between patients who received postoperative radiotherapy and those who did not clearly suggests the important role of radiation therapy in the treatment of PG-DLBCL. Validation of these results by future studies is necessary.

An important issue needs to be addressed. Positron emission tomography-CT is now the standard of care for staging of DLBCL and also important for defining the radiation fields with ISRT. However, in some developing countries such as People's Republic of China, positron emission tomography-CT is not widely available and some patients cannot afford it. Similarly, rituximab is expensive and is not covered by health insurance; so, patients who cannot afford it were prescribed the CHOP regimen. That is the significance of this study.

The limitations of this study are the relatively low average number of lymphadenectomies and small patient sample, which make the results less convincing, although the surgery was a standard D2 gastrectomy. Larger series are needed in the future.

In conclusion, the radiation field for patients with PG-DLBCL is largely dependent on the depth of invasion; as a result, primary endoscopic ultrasonography is highly recommended. Larger series and longer follow-up are needed to further optimize the radiation volumes for PG-DLBCL.

\section{Acknowledgments}

We thank H-Q L for her great help in revising the manuscript. The funding for the study was supported by the National Natural Science Foundation of China 81502659. This study was accepted as an oral presentation in the RSNA 101st Scientific Assembly and Annual Meeting, November 29 December 4, 2015, McCormick Place, Chicago, IL, USA. 


\section{Disclosure}

The authors report no conflicts of interest in this work.

\section{References}

1. Avilés A, Nambo MJ, Neri N, et al. The role of surgery in primary gastric lymphoma: results of a controlled clinical trial. Ann Surg. 2004;240(1): $44-50$.

2. Cheung MC, Housri N, Ogilvie MP, Sola JE, Koniaris LG. Surgery does not adversely affect survival in primary gastrointestinal lymphoma. J Surg Oncol. 2009;100(1):59-64.

3. Cirocchi R, Farinella E, Trastulli S, et al. Surgical treatment of primitive gastro-intestinal lymphomas: a systematic review. World J Surg Oncol. 2011;9:145.

4. Park YH, Lee SH, Kim WS, et al. CHOP followed by involved field radiotherapy for localized primary gastric diffuse large B-cell lymphoma: results of a multicenter phase II study and quality of life evaluation. Leuk Lymphoma. 2006;47(7):1253-1259.

5. Ishikura S, Tobinai K, Ohtsu A, et al. Japanese multicenter phase II study of CHOP followed by radiotherapy in stage I-II, diffuse large B-cell lymphoma of the stomach. Cancer Sci. 2005;96(6):349-352.

6. Koch P, Probst A, Berdel WE, et al. Treatment results in localized primary gastric lymphoma: data of patients registered within the German multicenter study (GIT NHL 02/96). J Clin Oncol. 2005;23(28): 7050-7059.

7. Kuo SH, Yeh KH, Wu MS, et al. Helicobacter pylori eradication therapy is effective in the treatment of early-stage $\mathrm{H}$ pylori-positive gastric diffuse large B-cell lymphomas. Blood. 2012;119(21):4838-4844.

8. Psyrri A, Papageorgiou S, Economopoulos T. Primary extranodal lymphomas of stomach: clinical presentation, diagnostic pitfalls and management. Ann Oncol. 2008;19:1992-1999.

9. Ferreri AJ, Cordio S, Ponzoni M, Villa E. Non-surgical treatment with primary chemotherapy, with or without radiation therapy, of stage I-II high-grade gastric lymphoma. Leuk Lymphoma. 1999;33(5-6): 531-541.

10. Ferreri AJ, Montalbán C. Primary diffuse large B-cell lymphoma of the stomach. Crit Rev Oncol Hematol. 2007;63(1):65-71.

11. Aleman BM, Haas RL, van der Maazen RW. Role of radiotherapy in the treatment of lymphomas of the gastrointestinal tract. Best Pract Res Clin Gastroenterol. 2010;24(1):27-34.

12. Illidge $T$, Specht $L$, Yahalom J, et al. Modern radiation therapy for nodal non-Hodgkin lymphoma-target definition and dose guidelines from the International Lymphoma Radiation Oncology Group. Int $J$ Radiat Oncol Biol Phys. 2014;89(1):49-58.

13. Swerdlow S, Campo E, Harris N et al. World Health Organization Classification of Tumors of Haematopoietic and Lymphoid Tissues. Lyon, France: WHO; 2008.

14. Hans CP, Weisenburger DD, Greiner TC, et al. Confirmation of the molecular classification of diffuse large B-cell lymphoma by immunohistochemistry using a tissue microarray. Blood. 2004;103(1): 275-282.

15. Rohatiner A, d'Amore F, Coiffier B, et al. Report on a workshop convened to discuss the pathological and staging classifications of gastrointestinal tract lymphoma. Ann Oncol. 1994;5(5):397-400.

OncoTargets and Therapy

\section{Publish your work in this journal}

OncoTargets and Therapy is an international, peer-reviewed, open access journal focusing on the pathological basis of all cancers, potential targets for therapy and treatment protocols employed to improve the management of cancer patients. The journal also focuses on the impact of management programs and new therapeutic agents and protocols on

Submit your manuscript here: http://www.dovepress.com/oncotargets-and-therapy-journal
16. Eisenhauer EA, Therasse P, Bogaerts J, et al. New response evaluation criteria in solid tumours: Revised RECIST guideline (version 1.1). Eur J Cancer. 2009;45(2):228-247.

17. Reyes F, Lepage E, Ganem G, et al; Groupe d'Etude des Lymphomes de l'Adulte (GELA). ACVBP versus CHOP plus radiotherapy for localized aggressive lymphoma. N Engl J Med. 2005;352(12):1197-1205.

18. Miller TP, Dahlberg S, Cassady JR, et al. Chemotherapy alone compared with chemotherapy plus radiotherapy for localized intermediate- and high-grade non-Hodgkin's lymphoma. N Engl J Med. 1998;339(1): $21-26$.

19. Horning SJ, Weller E, Kim K, et al. Chemotherapy with or without radiotherapy in limited-stage diffuse aggressive non-Hodgkin's lymphoma: Eastern Cooperative Oncology Group study 1484. JClin Oncol. 2004;22(15):3032-3038.

20. Miller TP, LeBlanc M, Spier C, et al. CHOP alone compared to CHOP plus radiotherapy for early stage aggressive non-Hodgkin's lymphomas: update of the Southwest Oncology Group (SWOG) randomized trial. Blood. 2001;98:724a.

21. Santos LVD, Lima J, Lima CSP, Sasse AD. Is there a role for consolidative radiotherapy in the treatment of aggressive and localized non-Hodgkin lymphoma? A systematic review with meta-analysis. BMC Cancer. 2012;12(1):288.

22. Bonnet C, Fillet G, Mounier N, et al. CHOP alone compared with CHOP plus radiotherapy for localized aggressive lymphoma in elderly patients: a study by the Groupe d'Etude des Lymphomes de l'Adulte. J Clin Oncol. 2007;25(7):787-792.

23. Liu GF, Bair RJ, Bair E, Liauw SL, Koshy M. Clinical outcomes for gastric cancer following adjuvant chemoradiation utilizing intensity modulated versus three-dimensional conformal radiotherapy. PLoS One. 2014;9(1):e82642.

24. Minn AY, Hsu A, La T, et al. Comparison of intensity-modulated radiotherapy and 3-dimensional conformal radiotherapy as adjuvant therapy for gastric cancer. Cancer. 2010;116(16):3943-3952.

25. Boda-Heggemann J, Hofheinz RD, Weiss C, et al. Combined adjuvant radiochemotherapy with IMRT/XELOX improves outcome with low renal toxicity in gastric cancer. Int J Radiat Oncol Biol Phys. 2009;75(4): $1187-1195$.

26. Lohr F, Dobler B, Mai S, et al. Optimization of dose distributions for adjuvant locoregional radiotherapy of gastric cancer by IMRT. Strahlenther Onkol. 2003;179(8):557-563.

27. Montalbán C, Castrillo JM, Abraira V, et al. Gastric B-cell mucosaassociated lymphoid tissue (MALT) lymphoma. Clinicopathological study and evaluation of the prognostic factors in 143 patients. Ann Oncol. 1995;6(4):355-362.

28. Eidt S, Stolte M, Fischer R. Factors influencing lymph node infiltration in primary gastric malignant lymphoma of the mucosa-associated lymphoid tissue. Pathol Res Pract. 1994;190(11):1077-1081.

29. Park W, Chang SK, Yang WI, et al. Rationale for radiotherapy as a treatment modality in gastric mucosa-associated lymphoid tissue lymphoma. Int J Radiat Oncol Biol Phys. 2004;58(5):1480-1486.

\section{Dovepress}

patient perspectives such as quality of life, adherence and satisfaction. The manuscript management system is completely online and includes a very quick and fair peer-review system, which is all easy to use. Visit http://www.dovepress.com/testimonials.php to read real quotes from published authors. 